\title{
Pregabalin on Hdac2 and Inpp5f levels in rats with CCI-induced neuropathic pain
}

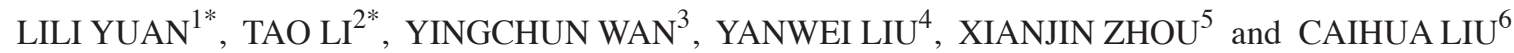 \\ ${ }^{1}$ Department of Anesthesiology, The Fifth Hospital of Wuhan, Wuhan, Hubei 430050; Departments of \\ ${ }^{2}$ Anesthesiology and ${ }^{3}$ Endocrinology, China-Japan Union Hospital of Jilin University, Changchun, Jilin 130033; \\ ${ }^{4}$ Department of General Surgery, Taihe Hospital, Shiyan, Hubei 442000; ${ }^{5}$ Department of Anesthesiology, \\ First Maternity and Infant Hospital Affiliated to Tongji University, Shanghai 200040; \\ ${ }^{6}$ Department of Anesthesiology, The Central Hospital of Wuhan, Tongji Medical College, \\ Huazhong University of Science and Technology, Wuhan, Hubei 430014, P.R. China
}

Received May 22, 2018; Accepted November 19, 2018

DOI: $10.3892 /$ etm.2018.7037

\begin{abstract}
Hdac2/Inpp5f on the therapeutic effect of pregabalin on neuropathic pain in chronic constriction injury (CCI) rats was investigated. A total of 90 healthy SD rats were selected and divided into the treatment group, the model control group and the normal control group with 30 rats in each group. Rats in the treatment and model control groups were used to construct CCI model. The control group was treated with sham surgery. The model control group was not treated after surgery. The treatment group was treated with pregabalin on the 8th day after operation. Behavioral experiments were performed to measure mechanical withdrawal threshold (MWT) and thermal withdrawal latency (TWL). The expression of inflammatory cytokines TNF $\alpha$ and IL-1 $\beta$ was detected by ELISA kit and expression of Hdac2 and Inpp5f mRNA was detected by RT-qPCR. Before treatment, compared with the normal control group, MWT and TWL values of the treatment and model control groups were significantly decreased $(\mathrm{P}<0.05)$. At different time-points after treatment, MWT and TWL were higher in the treatment group than in the model control group and were lower than in the normal control group. At 21 days after operation, the levels of TNF $\alpha$ and IL-1 $\beta$ in the model control and treatment groups were higher than those in the normal control group $(\mathrm{P}<0.05)$. The expression level of Hdac2 was higher in the treatment group than in the normal control group $(\mathrm{P}<0.05)$. The expression level of Inpp5f mRNA in the treatment and normal control groups was significantly higher than that in the model control group $(\mathrm{P}<0.05)$, but
\end{abstract}

Correspondence to: Dr Caihua Liu, Department of Anesthesiology, The Central Hospital of Wuhan, Tongji Medical College, Huazhong University of Science and Technology, 26 Shengli Street, Jiangan, Wuhan, Hubei 430014, P.R. China

E-mail: c4r249@163.com; 56834592@qq.com

\section{${ }^{*}$ Contributed equally}

Key words: Hdac2, Inpp5f, pregabalin, neuropathic pain, CCI rats the expression level of Inpp5f mRNA in the treatment group was lower than that in the normal control group $(\mathrm{P}<0.05)$. Therefore, pregabalin can effectively relieve neuropathic pain in CCI rats, and its efficacy is related to Hdac2 and Inpp5f. Our study provides reference for clinical treatment of neuropathic pain induced by CCI.

\section{Introduction}

Under normal circumstances, pain is a protective reaction in the body. Pain response can lead to a series of reflexes to avoid damage (1). However, in neuropathic pain, due to damage or disturbance in a certain part of the reflex, such as the sensory system and central nervous system, the pain threshold is reduced, resulting in over-sensitive pain sensation caused by various stimuli, and even without any external stimulus, spontaneous pain would also be produced. This continuous intense pain causes great pressure on patients and also brings a series of stress changes to the body (2). The expression levels of the inflammatory cytokines TNF $\alpha$ and IL-1 $\beta$ are significantly elevated in chronic constriction injury (CCI) models, whereas TNF $\alpha$ is neurotoxic in the central nervous system (3). Neuropathic pain can be caused by a variety of factors, such as infection and spinal cord injury. Incidence of neuropathic pain is as high as $7 \%$, but the specific pathogenesis is not yet clear, and there is currently no effective treatment (4).

Histone deacetylase 2 (Hdac2) is widely present in eukaryotic organisms and plays an important role in the proliferation and differentiation of cells and in the homeostasis of the body. Acetylation plays an irreplaceable role in inflammation and sensitization of chronic pain (5). Acetylation can open the chromatin structure, activate transcription sites, increase gene expression, and Hdac2 deacetylation can cause the polycondensation of chromatin, inhibit transcription of related genes, and promote the occurrence of neuropathic pain (6). Inpp5f (Sac2), namely inositol 4-phosphatase, has inositol tetraphosphate (PI4P) as its substrate. Phosphoinositides (PIs) are metabolites produced by the phosphorylation of phosphatidylinositol at the inositol $3,4,5$ positions. PIs are key regulatory phospholipids located in cell membranes, which can regulate 
cell signal transduction, membrane transport, cytoskeleton and lipid balance. Phosphorylation of PI4P affects the function of synaptic binding proteins which use PI4P as a substrate, thereby affecting the transport of synaptic vesicles to the synaptic active area and inhibiting the transmission of pain signals in neuropathic pain $(7,8)$.

Pregabalin can agonize $\gamma$-aminobutyric acid (GABA) receptors, thereby interrupting voltage-dependent calcium channels and inhibiting neurotransmitter release in synaptic vesicles. Pregabalin therefore does not exert anti-inflammatory effects and does not affect physiological pain (9). Clinically, pregabalin is often used for the treatment of pathological pain in peripheral nerves and for the adjuvant treatment of localized epilepsy (10). This study explored the effect of pregabalin on neuropathic pain in CCI rats by measuring the expression levels of Hdac2 and Inpp5f, so as to explore its pathogenesis and provide possible targets for related drugs.

\section{Materials and methods}

Materials. A total of 90 healthy, specific pathogen-free clean grade male Sprague-Dawley rats aged 2 months and weighing $200 \pm 20 \mathrm{~g}$ were provided by Shanghai Sleek Laboratory Animal Co., Ltd., Shanghai, China [SCXK (Shanghai) 2012-0002]. The rats were kept in cages with free access to food and water and temperature was maintained at $23^{\circ} \mathrm{C}$. There was a $12 / 12 \mathrm{~h}$ light/dark cycle and humidity was $60 \pm 10 \%$. A tactile pain measuring instrument was provided from Friends Honesty life Sciences Company Limited (Beijing, China). A tail-flick foot tester was purchased from Shanghai Yuyan Instruments Co., Ltd. (Shanghai, China). Pregabalin was provided by Shanghai Shifeng Biological Technology Co., Ltd. (Shanghai, China). A UV spectrophotometer MD1000 was purchased from Beijing Thmorgan Biotecnology Co., Ltd. (Beijing, China). TRIzol reagent was purchased from (Shanghai Mingjing Biotechnology Co., Ltd. (Shanghai, China). Reverse transcription kit was purchased from Thermo Fisher Scientific (China) Co., Ltd. (Shanghai, China). PCR kit was purchased from Beijing ComWin Biotech Co., Ltd. (Beijing, China). ELISA kit was provided by Wuhan Moshake Biotech Co., Ltd. (Wuhan, China) and Primer sequences were purchased from Sangon Biotech (Shanghai) Co., Ltd. (Shanghai, China).

The study was approved by the Ethics Committee of The Fifth Hospital of Wuhan (Wuhan, China).

Model establishment and grouping. SD rats were randomly divided into 3 groups: the treatment group, the model control group and the normal control group (30 in each group). The normal control group was treated with sham operation. The treatment and model control groups were subjected to CCI modeling based on Bennett method (11). Each rat was weighed and given an intraperitoneal injection of $10 \%$ chloral hydrate at a dose of $200 \mathrm{mg} / \mathrm{kg}$ for anesthesia. The surgical area was disinfected with $75 \%$ ethanol, and the skin parallel to the upper side of the femur was incised. The muscles and sciatic nerves were bluntly separated. The fork of the sciatic nerve was found and was ligated with $4-0$ silk thread at $5 \mathrm{~cm}$ above the site (the normal control group was not ligated after separating nerves). Four ligations with an interval of $1 \mathrm{~cm}$ were made. Ganglion subsidence but unblocked outer membrane blood flow was the standard. Incision was closed layer by layer and the muscles and skin were sutured. Injection of penicillin at a dose of 80,000 IU was performed. Mechanical withdrawal threshold (MWT) and thermal withdrawal latency (TWL) were measured 1 week after operation. MWT decline of $>40 \%$ was regarded as a successful modeling. Modeling was successful in 28 out of 30 rats $(93.33 \%)$ in the treatment group and in 27 out of 30 rats $(90.00 \%)$ in the model control group. Rats in the treatment group were weighed on the 8th day after operation and pregabalin was given by gavage at a dose of $3 \mathrm{mg} / \mathrm{kg}$. Rats in the model control group received the same volume of normal saline. MWT and TWL of the rats in the three groups were detected before and at approximately 10 am at 7, 10, 14, and 21 days after modeling. Behavioral test was completed and all rats were sacrificed by decapitation at 21 days after operation. Lumbar enlargement tissue was isolated from the lumbar spinal cord.

MWT assay. MWT of the rats in the three groups was detected before and at approximately 10 am at 7, 10, 14, and 21 days after modeling. Rats were placed on test bench for $30 \mathrm{~min}$ and a pressure sensor was used to prick the middle of the forefoot of rats vertically. The stimulation was performed slowly and uniformly to avoid disturbing rats. Paw withdrawal reactions and tail flick reactions were positive reaction, and the number of grams on the electronic display screen at this time-point was MWT. The left and right paws of rats were tested alternately and measurement was performed once every $5 \mathrm{~min}$ and was repeated three times. The average value was calculated.

TWL determination. TWL of the rats in three groups were detected before and at approximately 10 am at 7, 10, 14, and 21 days after modeling. The rats were placed in a plexiglass box for $15 \mathrm{~min}$ before the test. A halogen spotlight was used to vertically illuminate the hind paw of the rat. Leg withdrawing and reaction evading were positive reactions and the timing at this time-point was TWL. The left and right paws of the rats were tested alternately and measurement was performed once every $5 \mathrm{~min}$ and was repeated three times. The average value was calculated.

Detection of inflammatory factors by ELISA. Enlarged lumbar spinal cord tissues in each group were extracted to grind and homogenize and diluted 30-fold, and the content of inflammatory factors was detected by ELISA according to the manufacturer's instructions of TNF $\alpha$ and IL-1 $\beta$ ELISA.

Determination of Hdac2 and Inpp5f $m R N A$ by RT-qPCR. Enlarged lumbar spinal cord tissues were subjected to total RNA extraction using TRIzol reagent according to the manufacturer's instructions. A micro-ultraviolet spectrophotometer was used to measure the concentration and purity of SYBR-Green (Thermo Fisher Scientific, Inc., Waltham, MA, USA) and A260/A280 ratio should be between 1.8 and 2.0. Reverse transcription was performed using a $20 \mu \mathrm{l}$ reaction system in strict accordance with the manufacturer's instructions. Reaction conditions were: $37^{\circ} \mathrm{C}$ for $45 \mathrm{~min}$ and $95^{\circ} \mathrm{C}$ for $5 \mathrm{~min}$. The synthesized cDNA was performed using a $20 \mu \mathrm{l}$ amplification system in accordance with the instructions of RT-qPCR kit. Reaction conditions were: $95^{\circ} \mathrm{C}$ for $10 \mathrm{~min}$, 


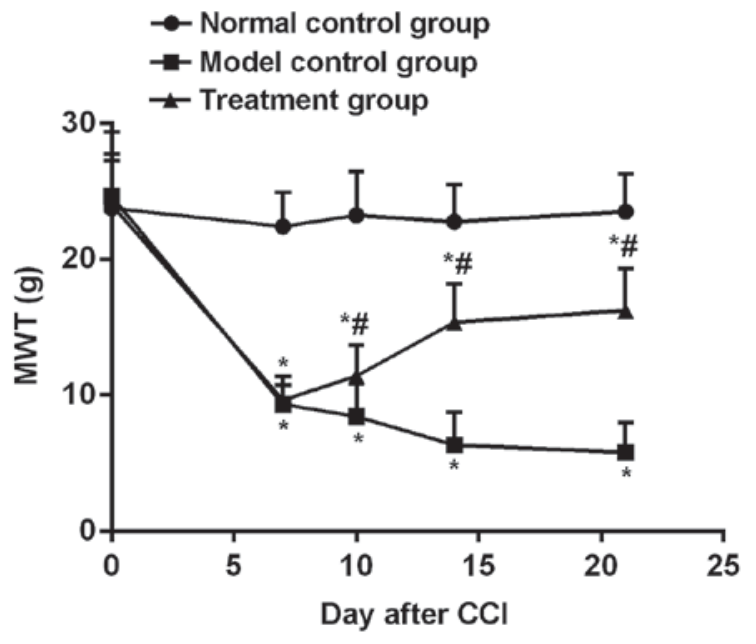

Figure 1. MWT changes in each group. Before the modeling, there was no significant difference in MWT among the normal control, model control and treatment groups $(\mathrm{P}>0.05)$. After 7 days, the treatment and model control groups showed a significantly lower MWT compared with the normal control group $(\mathrm{P}<0.05)$, and there was no difference in MWT between the treatment and model control groups $(\mathrm{P}>0.05)$. Intragastric administration of drug was performed at 8 days after operation. On the 10th, 14th and 21st days after modeling, MWT of the treatment group was higher than that of the model control group, but lower than that of the normal control group $(\mathrm{P}<0.05)$. MWT is at a relatively stable level in the normal control group at 7-21 days after operation, but MWT increased in the treatment group and decreased in the model control group. ${ }^{*} \mathrm{P}<0.05$, compared with the normal control group; ${ }^{\text {}} \mathrm{P}<0.05$, compared with the model control group.

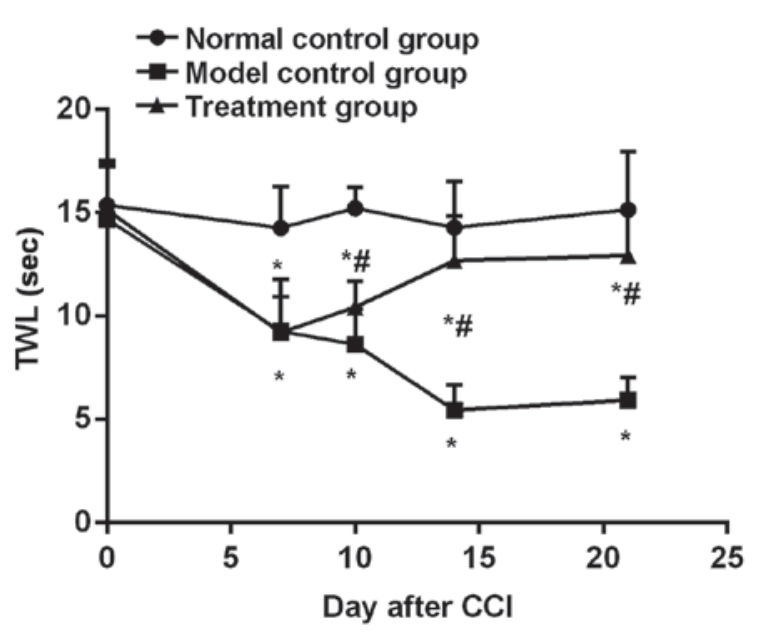

Figure 2. Changes of TWL in the rats of each group. Before modeling, TWL values of the normal control, model control, and treatment groups were $14.25 \pm 2.01,9.25 \pm 2.51$, and $9.19 \pm 1.76 \mathrm{~g}$, respectively, and there was no statistical difference in TWL among the three groups $(\mathrm{P}>0.05)$. At 7 days after operation, TWL gradually decreased in the model control group and increased gradually in the treatment group until the 21st day. Compared with the level to that at 7 days, TWL increased significantly at 21 days $(\mathrm{P}<0.01)$. TWL is always shorter in the treatment group than in the normal control group $(\mathrm{P}<0.05)$. ${ }^{\mathrm{P}} \mathrm{P}<0.05$, compared with the normal control group; ${ }^{*} \mathrm{P}<0.05$, compared with the model control group.

followed by 40 cycles of $95^{\circ} \mathrm{C}$ for $10 \mathrm{sec}, 60^{\circ} \mathrm{C}$ for $20 \mathrm{sec}$ and $72^{\circ} \mathrm{C}$ for $10 \mathrm{sec}$, and then $72^{\circ} \mathrm{C}$ for another $5 \mathrm{~min}$. Primers used in PCR reactions were: 5'-GTGGGGCGCCCCAGGCACCA-3' (forward) and 5'-CTCCTTAATGTCACGCACGATTTC-3' (reverse) for $\beta$-actin; 5'-TGACATTGTGCTTGCTGTCC-3' (forward) and 5'-CCCTCAAGTCTCCTGTTCCA-3' (reverse)
Table I. The content of TNF $\alpha$ and IL- $1 \beta$ in enlarged lumbar spinal cord tissues at 21 days after operation.

\begin{tabular}{lcc}
\hline Groups & TNF $\alpha(\mathrm{pg} / \mathrm{mg})$ & $\mathrm{IL}-1 \beta(\mathrm{pg} / \mathrm{mg})$ \\
\hline Normal control & $27.42 \pm 4.75$ & $55.12 \pm 9.13$ \\
Model control & $54.24 \pm 7.86^{\mathrm{a}}$ & $110.86 \pm 16.31^{\mathrm{a}}$ \\
Treatment & $39.42 \pm 6.32^{\mathrm{a}, \mathrm{b}}$ & $78.82 \pm 12.42^{\mathrm{a}, \mathrm{b}}$ \\
\hline
\end{tabular}

${ }^{\mathrm{a}} \mathrm{P}<0.05$ compared with the normal control group; ${ }^{\mathrm{b}} \mathrm{P}<0.05$ compared with the model control group.

for Hdac2; 5'-GGAGGCCACTTGTGTAGAT-3' (forward) and 5'-GGAGGCCACTTGTGTAGAT-3' (reverse) for Inpp5f. Data were processed using $2^{-\Delta \Delta C q}$ method (12).

Statistical analysis. Data were processed using SPSS 19.0 (Asia Analytics Formerly SPSS China) software package. MWT and TWL values, and the content or expression of related substances were expressed as mean \pm standard deviation. According to data distribution characteristics, comparisons among three or more groups were compared by analysis of variance followed by Dunnett's test as a post hoc test. Intragroup comparisons were performed by repeated measurement analysis of variance. The significance level was $\alpha=0.05$.

\section{Results}

Changes in MWT of rats in each group. Before modeling, MWT values of the normal control, model control and treatment groups were $23.76 \pm 3.51,24.65 \pm 4.73$, and $24.01 \pm 3.76 \mathrm{~g}$, respectively, and no significant differences were found among the three groups $(\mathrm{P}>0.05)$. At 7 days after modeling, MWT values of the normal control, model control and treatment groups were $22.41 \pm 2.51,9.34 \pm 1.42$, and $9.63 \pm 1.76 \mathrm{~g}$, respectively. MWT of the treatment and model control groups was lower than that of the normal control group $(\mathrm{P}<0.05)$. There was no difference in the MWT between the treatment and model control groups $(\mathrm{P}>0.05)$. Intragastric administration of drug was performed at 8 days after operation. On the 10th, 14th and 21st days after modeling, MWT of the treatment group was higher than that of the model control group, but lower than that of the normal control group $(\mathrm{P}<0.05)$. MWT was at a relatively stable level in the normal control group at 7-21 days after operation (Fig. 1).

Changes in TWL of rats in each group. Before modeling, TWL values of the normal control, model control and treatment groups were $15.35 \pm 2.15,14.68 \pm 2.52$, and $15.12 \pm 2.21 \mathrm{~g}$, respectively, and there was no statistical difference in TWL among the three groups $(\mathrm{P}>0.05)$. At 7 days after operation, values of MWT in the normal control, model control and treatment groups were $14.25 \pm 2.01,9.25 \pm 2.51$, and $9.19 \pm 1.76 \mathrm{~g}$, respectively. After operation, TWL gradually decreased in the model control group and increased gradually in the treatment group until the 21st day. Compared with the level to that at 7 days, TWL increased significantly at 21 days $(\mathrm{P}<0.01)$. TWL was always shorter in the treatment group than in the normal control group (Fig. 2). 


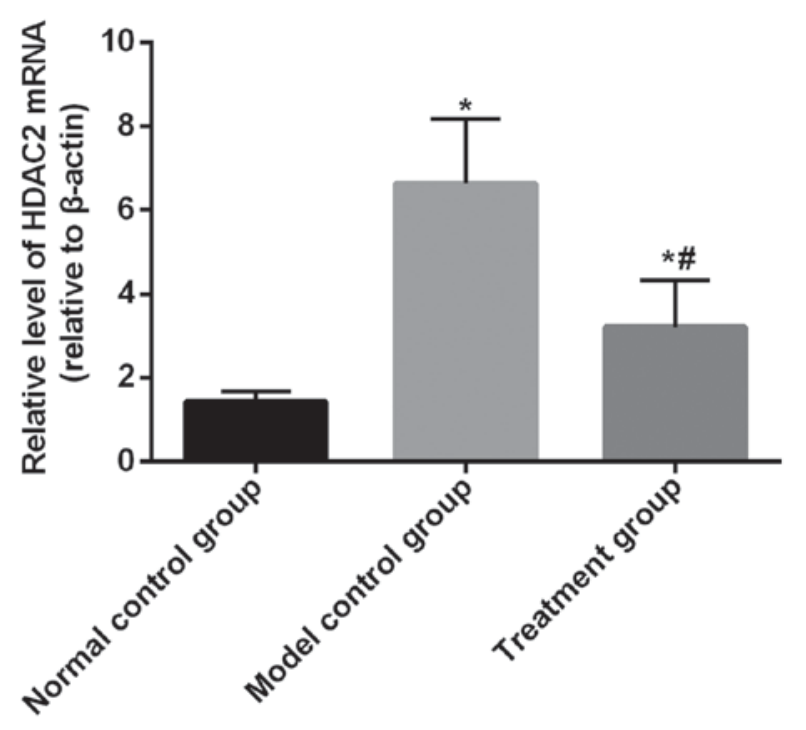

Figure 3. HDAC2 expression detected by RT-qPCR. At 21 days, the expression of HDAC2 and Inpp5f mRNA in lumbar enlargement tissues of each group was detected. The expression levels of HDAC2 in the normal control and treatment groups were lower than those in the model control group. The expression levels of HDAC2 in the treatment group were higher than that in the normal control group $(\mathrm{P}<0.05)$. ${ }^{*} \mathrm{P}<0.05$, compared with the normal control group; ${ }^{\#} \mathrm{P}<0.05$, compared with the model control group.

ELISA detection of TNF $\alpha$ and IL-1 $\beta$. At 21 days after operation, TNF $\alpha$ and IL-1 $\beta$ contents in enlarged lumbar spinal cord tissues from each group were measured. The levels of TNF $\alpha$ and IL-1 $\beta$ in the model control and treatment groups were higher than those in the normal control group. The levels of TNF $\alpha$ and IL-1 $\beta$ in the treatment group were lower than those in the model control group $(\mathrm{P}<0.05)$ (Table I).

Determination of Hdac2 and Inpp5f mRNA by RT-qPCR. The expression of Hdac 2 and Inpp5f mRNA was detected in lumbar enlargement tissues of each group at 21 days after operation. The expression level of Hdac 2 in the normal control group $(1.43 \pm 0.24)$ and treatment group $(3.21 \pm 1.11)$ was significantly lower than that in the model control group (6.64 \pm 1.54$)$. The expression level of Hdac2 in the treatment group was significantly higher than that in the normal control group $(\mathrm{P}<0.05$, Fig. 3). The expression level of Inpp5f mRNA in the treatment group $(0.75 \pm 0.21)$ and normal control group $(1.37 \pm 0.26)$ was significantly higher than that in the model control group $(0.42 \pm 0.06, \mathrm{P}<0.05)$, but the expression level of Inpp5f mRNA was lower in the treatment group than in the normal control group $(\mathrm{P}<0.05$, Fig. 4).

\section{Discussion}

Neuropathic pain is the pain sensitization resulting from nervous system injury caused by a variety of complex factors and spontaneous pain is produced, which brings great harm to both individuals and society (13). This disease involves a wide variety of complex neural pathways and transmitters, therefore, up to now, its pathogenesis remains unclear and there is no effective therapeutic regimen (14). Hdac2 is mainly expressed in post-mitotic or differentiated neurons. In addition to the actions on histones, Hdac 2 can also act on non-histone proteins,

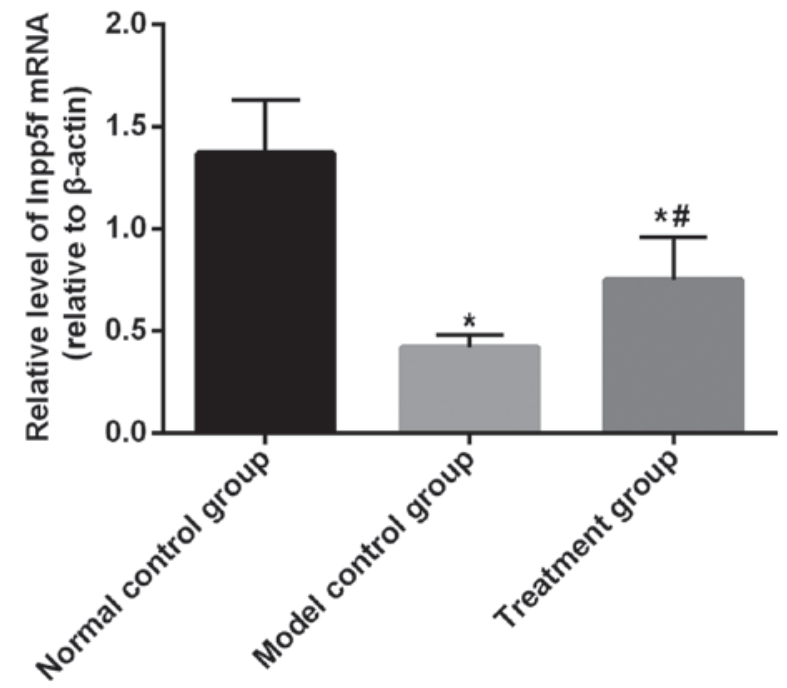

Figure 4. The expression of Inpp5f detected by RT-qPCR. The expression level of Inpp5f mRNA in the treatment and normal control groupa was significantly higher than that in the model control group $(\mathrm{P}<0.05)$, but the expression level of Inpp5f mRNA in the treatment group was lower than that in the normal control group $(\mathrm{P}<0.05)$. ${ }^{*} \mathrm{P}<0.05$, compared with the normal control group; ${ }^{\#} \mathrm{P}<0.05$, compared with the model control group.

such as glutamic acid decarboxylase 65 , thereby inhibiting GABA function and improving neuropathic pain (15). The protein encoded by Inpp5f has a SAC phosphatase domain, so Inpp5f can exert SAC phosphatase activity, inhibit the conversion of PIP2 to PIP3, promote the conversion of PIP2 to PIP, and inhibit PI3K/AKT signaling pathway (16).

Nakatsu et al (17) found that the Sac domain of synaptojanin has Inpp5f activity, which may be related to the release of synaptic proteins that regulate neurotransmitters and their involvement in the early development of neurons. Zou et al (18) found that neurons lacking Inpp5f were able to regenerate axons more efficiently than wild-type neurons. Zhang et al (19) found that silencing of Inpp5f (Sac2) resulted in regeneration of axons and increased growth cones. Pregabalin reduces intracellular calcium concentration by inhibiting voltage-dependent calcium channels. Pregabalin can prevent excitatory neurotransmitters from acting on the postsynaptic membrane, block the production of neuropathic pain, and can effectively treat allergy of nervous system to pain as well as hypersensitivity symptoms (10). Damaged nerve can induce $\mathrm{TNF} \alpha$ and IL- $1 \beta$ to express in ganglion, TNF $\alpha$ and IL-1 $\beta$ can further promote the occurrence of inflammation, and can also induce the release of excitatory neurotransmitters, resulting in sensitized nerve (20).

No significant differences in MWT were found among the 3 groups before modeling. At 7 days after modeling, the treatment and model control groups showed a significantly lower MWT (a reduction range of $>40 \%$ ) compared with the normal control group, indicating that the modeling was successful. At 7-21 days, MWT in the normal control group was at a relatively stable level, but MWT increased in the treatment group and decreased in the model control group; but the lowest level was always observed in the normal control group, followed by the treatment group and then the model control group. The increase amplitude of MWT at 14-21 days in the treatment group decreased, indicating that pregabalin can inhibit 
the decrease of MWT in CCI rats, but cannot completely reverse the changes and this effect has a certain timeliness. No significant differences in TWL were found among the 3 groups before modeling. After 7 days, TWL of the model control group gradually decreased, and TWL of the treatment group gradually increased until 14 days. TWL in the treatment group was always smaller than that of the normal control group, indicating that pregabalin has an inhibitory effect on the decrease of TWL in CCI rats. At 21 days after operation, the levels of TNF $\alpha$ and IL-1 $\beta$ in lumbar enlargement tissue of the model control and treatment groups were higher than those in the normal control group, and levels of TNFa and IL-1 $\beta$ in the treatment group were lower than those in the model control group. It indicated that the contents of TNFo and IL-1 $\beta$ in rat spinal cord increased after CCI modeling, and $\mathrm{TNF} \alpha$ and IL-1 $\beta$ may be involved in the pathogenesis of CCI. Khan et al (21) also reached the same conclusion. Although pregabalin does not exert anti-inflammatory effects, its effect on repairing nervous system damage in rats may inhibit the rise in TNF $\alpha$ and IL-1 $\beta$ levels. At 21 days, the expression of Hdac2 and Inpp5f mRNA in lumbar enlargement tissues of each group was detected. The expression level of Hdac2 in the normal control and treatment groups was lower than that in the model control group. The expression level of Hdac2 was higher in the treatment group than in the normal control group. Yuan et al (22) also found that expression level of Hdac2 increased after nerve damage. This may be because Hdac2 can inhibit GABA-mediated inhibition of the endogenous pain pathways. After modeling, the expression of Hdac2 in the model control and treatment groups increased, and the inhibition of the endogenous pain pathway was weakened, and MWT and TWL values of the rats decreased. Pubbarenin can inhibit the activity of GABA receptors to counteract the inhibitory effect of Hdac2 on GABA, and the decrease of Hdac2 expression in the treatment group also reduces the decrease amplitude of MWT and TWL. The expression level of Inpp5f mRNA in the treatment and normal control groups was significantly higher than that in the model control group $(\mathrm{P}<0.05)$, but the expression level of Inpp5f mRNA in the treatment group was lower than that in the normal control group $(\mathrm{P}<0.05)$. Therefore, it is suggested that the expression of Inpp5f may be involved in the pathogenesis of neuropathic pain. Zou et al (18) found that Inpp5f can inhibit the repair of spinal cord injury, but its effect on neuropathic pain is unknown. PIP2 can upregulate TRPM8 receptors in dorsal root ganglion, so as to promote pain sensitization and participate in neuropathic pain. Inpp5f dephosphorylates PIP2 into PIP, so as to inhibit the formation of hyperalgesia, and thus alleviates the decline in MWT and TWL values in the treatment group (18). Trivedi et al (23) found that HDAC2 acts as a transcriptional repressor and can inhibit the activity of phosphatases such as PI3K and Inpp5f.

CCI rat model used in this experiment does not fully represent the actual occurrence of neuropathic pain, and in particular, it does not represent the hyperalgesia caused by genetic mutations. Mutations of Hdac2 and Inpp5f gene caused by viral infection or other autologous diseases also result in neuropathic pain (24). In our study, altered expression of Hdac2 and Inpp5f and values of MWT and TWL were observed in CCI rats, while the mechanism of the role of Hdac2 and Inpp5f in neuropathic pain remains unclear, or this may be because the decrease of MWT and TWL values leads to change of Hdac2/Inpp5f expression. Moreover, Pregabalin treatment may relieve neuropathic pain through Hdac2/Inpp5f or other pathways. Therefore, more studies are still needed to answer these questions.

In summary, Hdac2/Inpp5f may modulate neuropathic pain in rats. Pregabalin may effectively relieve neuropathic pain in CCI rats, and its action may be related to Hdac2/ Inpp5f. Therefore, our study may provide references for the clinical treatment of neuropathic pain.

\section{Acknowledgements}

Not applicable.

\section{Funding}

No funding was received.

\section{Availability of data and materials}

The datasets used and/or analyzed during the present study are available from the corresponding author on reasonable request.

\section{Authors' contributions}

LY and TL helped with model establishment and grouping. YW and YL helped with MWT assay. XZ performed ELISA. LY, TL and CL were responsible for RT-qPCR. All authors read and approved the final manuscript.

\section{Ethics approval and consent to participate}

The study was approved by the Ethics Committee of The Fifth Hospital of Wuhan (Wuhan, China).

\section{Patient consent for publication}

Not applicable.

\section{Competing interests}

The authors declare that they have no competing interests.

\section{References}

1. Bouhassira D and Attal N: Translational neuropathic pain research: A clinical perspective. Neuroscience 338: 27-35, 2016.

2. Finnerup NB, Haroutounian S, Kamerman P, Baron R, Bennett DL, Bouhassira D, Cruccu G, Freeman R, Hansson P, Nurmikko T, et al: Neuropathic pain: An updated grading system for research and clinical practice. Pain 157: 1599-1606, 2016.

3. Wu FX, Bian JJ, Miao XR, Huang SD, Xu XW, Gong DJ, Sun YM, Lu ZJ and Yu WF: Intrathecal siRNA against Toll-like receptor 4 reduces nociception in a rat model of neuropathic pain. Int J Med Sci 7: 251-259, 2010.

4. Lefaucheur JP: Cortical neurostimulation for neuropathic pain: State of the art and perspectives. Pain 157 (Suppl 1): S81-S89, 2016.

5. Chen D, Weng Y, Ouyang B, Guo M and Guo Q: Effect of intrathecal ropivacaine on spinal HDAC1 and HDAC 2 expression in rats with neuropathic pain. Chin J Anaesth 35: 1093-1095, 2015 (In Chinese). 
6. Maiarù M, Morgan OB, Tochiki KK, Hobbiger EJ, Rajani K, Overington DW and Geranton SM: Complex regulation of the regulator of synaptic plasticity histone deacetylase 2 in the rodent dorsal horn after peripheral injury. J Neurochem 138 222-232, 2016

7. Bai D, Zhang Y, Shen M, Sun Y, Xia Q, Zhang Y, Liu X, Wang H and Yuan L: Hyperglycemia and hyperlipidemia blunts the Insulin-Inpp5f negative feedback loop in the diabetic heart. Sci Rep 6: 22068, 2016.

8. Palermo G, Maisel D, Barrett M, Smith H, Duchateau-Nguyen G, Nguyen T, Yeh RF, Dufour A, Robak T, Dornan D, et al; REACH investigators: Gene expression of INPP5F as an independent prognostic marker in fludarabine-based therapy of chronic lymphocytic leukemia. Blood Cancer J 5: e353, 2015.

9. Toth C: Pregabalin: Latest safety evidence and clinical implications for the management of neuropathic pain. Ther Adv Drug Saf 5: 38-56, 2014

10. Verma V, Singh N and Singh Jaggi A: Pregabalin in neuropathic pain: Evidences and possible mechanisms. Curr Neuropharmacol 12: 44-56, 2014.

11. Bennett GJ and Xie YK: A peripheral mononeuropathy in rat that produces disorders of pain sensation like those seen in man. Pain 33: 87-107, 1988.

12. Livak KJ and Schmittgen TD: Analysis of relative gene expression data using real-time quantitative PCR and the 2(-Delta Delta C(T)) method. Methods 25: 402-408, 2001

13. Jiang BC, Cao DL, Zhang X, Zhang ZJ, He LN, Li CH, Zhang WW, Wu XB, Berta T, Ji RR, et al: CXCL13 drives spinal astrocyte activation and neuropathic pain via CXCR5. J Clin Invest 126: 745-761, 2016

14. Kim J, Ryu SB, Lee SE, Shin J, Jung HH, Kim SJ, Kim KH and Chang JW: Motor cortex stimulation and neuropathic pain: How does motor cortex stimulation affect pain-signaling pathways? J Neurosurg 124: 866-876, 2016.

15. Wang SE, Ko SY, Jo S, Choi M, Lee SH, Jo HR, Seo JY, Lee SH, Kim YS, Jung SJ, et al: TRPV1 regulates stress responses through HDAC2. Cell Rep 19: 401-412, 2017.

16. Kim HS, Li A, Ahn S, Song H and Zhang W: Inositol Polyphosphate-5-Phosphatase F (INPP5F) inhibits STAT3 activity and suppresses gliomas tumorigenicity. Sci Rep 4: 7330, 2014.
17. Nakatsu F, Messa M, Nández R, Czapla H,Zou Y, Strittmatter SM and De Camilli P: Sac2/INPP5F is an inositol 4-phosphatase that functions in the endocytic pathway. J Cell Biol 209: 85-95, 2015.

18. Zou Y, Stagi M, Wang X, Yigitkanli K, Siegel CS, Nakatsu F, Cafferty WB and Strittmatter SM: Gene-silencing screen for mammalian axon regeneration identifies Inpp5f (Sac2) as an endogenous suppressor of repair after spinal cord injury. J Neurosci 35: 10429-10439, 2015.

19. Zhang S, Sarmiere PD, Doolen S, Kluge B, Huang F, White JT and Holmberg E: Scar ablation combined with cell transplantation promotes tissue repair and axon regeneration in chronic contusion injury of rat spinal cord. 25th Annual NationalNeurotrauma-Society Symposium 24: 1256, 2007.

20. Lu Y, Jiang BC, Cao DL, Zhang ZJ, Zhang X, Ji RR and Gao YJ: TRAF6 upregulation in spinal astrocytes maintains neuropathic pain by integrating TNF- $\alpha$ and IL-1 $\beta$ signaling. Pain 155: 2618-2629, 2014

21. Khan J, Noboru N, Young A and Thomas D: Pro- and antiinflammatory cytokine levels (TNF- $\alpha$, IL-1 $\beta$, IL-6 and IL-10) in rat model of neuroma. Pathophysiology 24: 155-159, 2017.

22. Yuan Y, Peng W, Liu Y and Xu Z: Palmatine attenuates isoproterenol-induced pathological hypertrophy via selectively inhibiting HDAC2 in rats. Int J Immunopathol Pharmacol 30: 406-412, 2017

23. Trivedi CM, Luo Y, Yin Z, Zhang M, Zhu W, Wang T, Floss T, Goettlicher M, Noppinger PR, Wurst W, et al: Hdac2 regulates the cardiac hypertrophic response by modulating Gsk3 beta activity. Nat Med 13: 324-331, 2007.

24. Ma H, Marti-Gutierrez N, Park SW, Wu J, Lee Y, Suzuki K, Koski A, Ji D, Hayama T, Ahmed R, et al: Correction of a pathogenic gene mutation in human embryos. Nature 548: 413-419, 2017.

This work is licensed under a Creative Commons Attribution-NonCommercial-NoDerivatives 4.0 International (CC BY-NC-ND 4.0) License. 